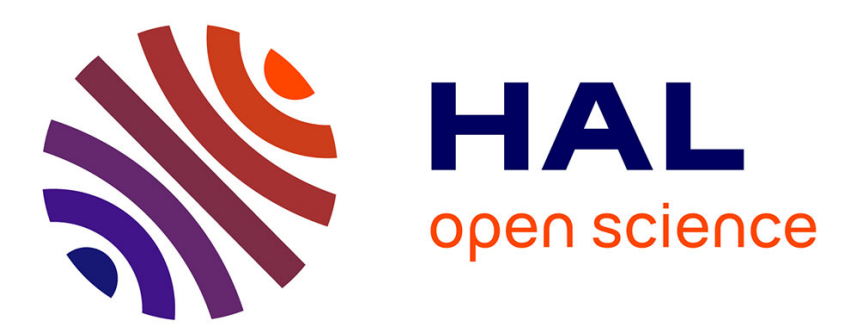

\title{
A viscosity solution method for the spreading speed formula in slowly varying media
}

François Hamel, Grégoire Nadin, Lionel Roques

\section{To cite this version:}

François Hamel, Grégoire Nadin, Lionel Roques. A viscosity solution method for the spreading speed formula in slowly varying media. Indiana University Mathematics Journal, 2011, 60 (4), pp.1229 1248. 10.1512/iumj.2011.60.4370 . hal-01080131

\section{HAL Id: hal-01080131 \\ https://hal.sorbonne-universite.fr/hal-01080131}

Submitted on 1 Mar 2016

HAL is a multi-disciplinary open access archive for the deposit and dissemination of scientific research documents, whether they are published or not. The documents may come from teaching and research institutions in France or abroad, or from public or private research centers.
L'archive ouverte pluridisciplinaire HAL, est destinée au dépôt et à la diffusion de documents scientifiques de niveau recherche, publiés ou non, émanant des établissements d'enseignement et de recherche français ou étrangers, des laboratoires publics ou privés. 


\title{
A viscosity solution method for the spreading speed formula in slowly varying media
}

\author{
François Hamel ${ }^{\mathrm{a}}$, Grégoire Nadin ${ }^{\mathrm{b}}$ and Lionel Roques ${ }^{\mathrm{c} *}$ \\ a Aix-Marseille Université \& Institut Universitaire de France
}

LATP, UMR 6632, Avenue Escadrille Normandie-Niemen, F-13397 Marseille Cedex 20, France

b CNRS, UMR 7598, Laboratoire Jacques-Louis Lions, 4 place Jussieu, F-75005, Paris, France

${ }^{\mathrm{c}}$ UR 546 Biostatistique et Processus Spatiaux, INRA, F-84000 Avignon, France

\begin{abstract}
In this paper, we consider reaction-diffusion-advection equations in slowly periodically oscillating media. We prove the existence of and we give explicit expressions of the asymptotic spreading speeds of invasion of the unstable state 0 in any direction, when the period of the invaded medium becomes infinitely large. The limiting spreading speeds involve families of 1-periodic Hamilton-Jacobi equations. In the case of one-dimensional reaction-diffusion equations, we analyze the relative effects of small perturbations of the diffusion and the reaction coefficients, and we compare the spreading speeds in slowly oscillating media to the homogenized spreading speeds in rapidly oscillating media.
\end{abstract}

\section{Introduction}

In this paper, we are concerned with spreading properties of the solutions of reactiondiffusion equations of the type

$$
\frac{\partial u}{\partial t}=\operatorname{div}\left(a_{L}(x) \nabla u\right)+b_{L}(x) \cdot \nabla u+f_{L}(x, u), \quad t>0, \quad x=\left(x_{1}, \ldots, x_{N}\right) \in \mathbb{R}^{N}
$$

in slowly oscillating periodic media. That is, we assume that

$$
a_{L}(x)=a\left(\frac{x}{L}\right), \quad b_{L}(x)=b\left(\frac{x}{L}\right), \quad f_{L}(x, s)=f\left(\frac{x}{L}, s\right)
$$

*The authors are supported by the French "Agence Nationale de la Recherche" within the projects ColonSGS (first and third authors), PREFERED, and URTICLIM (third author). The first author is also indebted to the Alexander von Humboldt Foundation for its support. 
for all $x \in \mathbb{R}^{N}$ and $s \geq 0$, where the diffusion, drift and reaction coefficients

$$
a=\left(a_{i j}\right)_{1 \leq i, j \leq N}: \mathbb{R}^{N} \rightarrow S_{N}(\mathbb{R}), \quad b=\left(b_{i}\right)_{1 \leq i \leq N}: \mathbb{R}^{N} \rightarrow \mathbb{R}^{N} \text { and } f(\cdot, s): \mathbb{R}^{N} \rightarrow \mathbb{R}
$$

are 1-periodic, for short, that is they are 1-periodic in all variables $x_{1}, \ldots, x_{N}$. The notation $S_{N}(\mathbb{R})$ stands for the set of $N \times N$ symmetric matrices with real entries. We establish asymptotic limits as $L \rightarrow+\infty$ of some fundamental spreading quantities associated with the large-time behavior of the nonnegative solutions. Throughout the paper, the function $f$ is assumed to be such that

$$
f(x, 0)=0
$$

for all $x \in \mathbb{R}^{N}$, that is 0 is a steady state for equation (1). Under some conditions to be detailed later, the state 0 will be unstable and equation (1) also admits a unique positive stable steady state. One of the main questions addressed in this paper is to know at which speed the positive steady state invades the state 0 when the underlying medium is slowly varying, that is when $L$ is large.

In homogeneous media, the study of the spreading properties of such equations has begun with the pioneering papers by Fisher [15] and Kolmogorov, Petrovsky and Piskunov [28] in dimension 1, and later by Aronson and Weinberger [1] in any dimension. Since then, these equations have found applications in various domains such as combustion $[42,43]$ and population ecology $[38,40]$. But since the environment is generally far from being homogeneous, it is therefore natural to try to understand the effects of the spatial heterogeneities on the spreading properties of the solutions of equations of the type (1). This issue has been investigated in a recent but large literature, in periodic media (see e.g. $[6,16,17,18,30,41]$ for nonnegative and compactly supported initial conditions, see also $[31,34,41]$ for front-like initial data), in random stationary ergodic environments (see $[18,29,36,37]$ ), as well as in general heterogeneous media (see $[5,6,8,10]$ ).

The spreading speeds of invasion of the steady state 0 are characterized as follows. Assuming that all terms in (1) are $L$-periodic in $x$ and that the reaction term is differentiable with respect to its second variable at 0 and satisfies the KPP assumption

$$
f_{L}(\cdot, s) \leq \frac{\partial f_{L}}{\partial s}(\cdot, 0) \times s \text { for all } s \geq 0
$$

and under further assumptions on $a_{L}, b_{L}$ and $f_{L}$ (see the comment before Proposition 2.1 below), it is known $[6,16,41]$ that, given any nonzero compactly supported and nonnegative initial condition $u_{0}(x)$ and given any direction $e$ of the unit sphere $\mathbb{S}^{N-1}$, the solution $u(t, x)$ of the Cauchy problem (1) with initial condition $u_{0}$ propagates in the direction $e$ with a positive spreading speed $w_{L}^{*}(e)>0$ in the sense that

$$
\left\{\begin{aligned}
\lim _{t \rightarrow+\infty} u(t, x+c t e) & =0 \text { for all } c>w_{L}^{*}(e), \\
\liminf _{t \rightarrow+\infty} u(t, x+c t e) & >0 \text { for all } 0 \leq c<w_{L}^{*}(e),
\end{aligned} \quad \text { locally uniformly in } x \in \mathbb{R}^{N} .\right.
$$

When problem (1) admits a (unique) positive periodic steady state $p_{L}$, the spreading speed $w_{L}^{*}(e)$ in a given direction $e$ can be expressed in terms of the minimal speeds of 
pulsating fronts connecting 0 to $p_{L}$ and propagating in the directions having a positive inner product with $e$, see $[6,41]$. In the particular one-dimensional case, the spreading speeds in the directions +1 and -1 are then equal to the minimal speeds of pulsating fronts connecting 0 to $p_{L}$ and propagating in these directions. For further existence, uniqueness and stability results of KPP pulsating fronts in one-dimensional or higher-dimensional periodic media, we refer to $[4,7,9,20,22,31,34]$.

On the one hand, the homogenization limit of rapidly oscillating media, corresponding to small values of $L$, has been well studied. In this case, an explicit formula has been established for $w_{0}^{*}( \pm 1)=\lim _{L \rightarrow 0} w_{L}^{*}( \pm 1)$ when $N=1$ and the drift $b$ is constant, or more generally when $b$ is divergence-free in higher dimensions [12, 26, 33] (see also [11, 23, 24] for other nonlinearities). The dependence of the spreading speed with respect to the parameter $L$ has also been studied and it is also known that, for all directions $e$, the spreading speeds $w_{L}^{*}(e)$ are nondecreasing with respect to $L$, when $b$ is divergence-free (see [33] and numerical results in $[27,40]$ ).

On the other hand, for equations of the type (1) with KPP nonlinearities $f_{L}$, the question of the asymptotics of the spreading speeds $w_{L}^{*}(e)$ in slowly oscillating media, that is as $L \rightarrow+\infty$, had only been investigated in a recent work [21], in the one-dimensional case, for constant values of $a_{L}$ and spatially piecewise-constant reaction terms $f_{L}$. In the general heterogeneous framework investigated here, proving the existence of a limit for the spreading speeds $w_{L}^{*}(e)$ and obtaining a formula for $w_{\infty}^{*}(e):=\lim _{L \rightarrow \infty} w_{L}^{*}(e)$ in any direction $e$ was still an open problem. This question is solved in the present paper. Furthermore, in the one-dimensional case, we prove a formula for $w_{\infty}^{*}( \pm 1)$, which is expressed in terms of some integral quantities involving the underlying coefficients.

To our knowledge, apart from the above-mentioned reference [21], comparable problems have only been studied in some papers by Freidlin [17] and Evans and Souganidis [14]. These papers were concerned with the limiting Cauchy problems obtained after rescaling, when the space and time variables are of order $L$ and when the period $L$ becomes infinitely large. However, the representation formula they provided for the limiting spreading speeds $w_{L}^{*}(e)$, based on game theory techniques, is quite complicated (we also refer to $[19,43]$ for spreading properties of bistable equations in slowly oscillating media).

As a matter of fact, our new results about the limiting spreading speeds have interesting consequences regarding the effects of spatial heterogeneities. Firstly, when the functions $L \mapsto w_{L}^{*}(e)$ are non-decreasing - this is true if $b$ is divergence-free [33]- the limiting spreading speeds $w_{\infty}^{*}(e)$ provide upper bounds for the spreading speeds $w_{L}^{*}(e)$ in $L$-periodic media, which are more precise than earlier results given in [7], and the quantities $w_{\infty}^{*}(e)-w_{0}^{*}(e)$ give a measure of the maximal effect of the medium oscillations. Secondly, the formula for $w_{\infty}^{*}(e)$ which we prove in this paper for general equations (1) sheds light on the effect of diffusion compared to that of reaction in heterogeneous media. Namely, it shows that the spreading speeds can be more sensitive to perturbations of the diffusion coefficient than to perturbations of the reaction term. Thirdly, spreading properties of equation (1) in slowly oscillating media are related to the spreading properties of this equation in the limit of large reaction terms. We obtain the exact asymptotics of the spreading speeds in periodic media with large amplitude reaction terms as a by-product of our formula for $w_{\infty}^{*}(e)$. 
To obtain this formula for $w_{\infty}^{*}(e)$, we use the variational characterization which was first derived in [18] and later generalized in $[6,41]$ in more general media, that is

$$
w_{L}^{*}(e)=\min _{\lambda \in \mathbb{R}^{N}, \lambda \cdot e>0} \frac{k_{L}(\lambda)}{\lambda \cdot e},
$$

where, for $\lambda \in \mathbb{R}^{N}, k_{L}(\lambda)$ denotes the periodic principal eigenvalue of an elliptic operator defined below. The core of our proof relies on a viscosity solution method together with several qualitative and quantitative properties of the function $(\lambda, L) \mapsto k_{L}(\lambda)$.

\section{Hypotheses and main results}

\subsection{Assumptions}

Before stating our main results, let us make more precise the assumptions and notations used throughout this paper. One is given a symmetric matrix field $a=\left(a_{i j}\right)_{1 \leq i, j \leq N}$ of class $W^{1, \infty}\left(\mathbb{R}^{N} ; S_{N}(\mathbb{R})\right)$ (in particular, the coefficients $a_{i j}$ can be assumed to be continuous), a continuous advection field $b: \mathbb{R}^{N} \rightarrow \mathbb{R}^{N}$ and a continuous growth term $c: \mathbb{R}^{N} \rightarrow \mathbb{R}$, which are 1-periodic in $x$, that is

$$
a(x+k)=a(x), \quad b(x+k)=b(x), \quad c(x+k)=c(x) \text { for all } k \in \mathbb{Z}^{N} \text { and } x \in \mathbb{R}^{N} .
$$

The case when the coefficients are $L_{i}$-periodic with respect to each variable $x_{i}$, with $L_{i}>0$, can be reduced to the above assumption (5), after rescaling the variables. Throughout the paper, we assume that the matrices $a(x)$ are uniformly positive definite, that is, there exists $\nu>0$ such that

$$
a(x) \geq \nu I_{N} \text { for all } x \in \mathbb{R}^{N}
$$

in the sense of symmetric matrices, where $I_{N}$ denotes the identity matrix of size $N \times N$. We also assume that

$$
M:=\max _{x \in \mathbb{R}^{N}}\left(c(x)-\frac{b(x) a(x)^{-1} b(x)}{4}\right)>0,
$$

where, for any vectors $\xi=\left(\xi_{1}, \ldots, \xi_{N}\right)$ and $\xi^{\prime}=\left(\xi_{1}^{\prime}, \ldots, \xi_{N}^{\prime}\right)$ in $\mathbb{R}^{N}$ and any symmetric matrix $A=\left(A_{i j}\right)_{1 \leq i, j \leq N}$, the quantity $\xi A \xi^{\prime}$ denotes

$$
\xi A \xi^{\prime}=\sum_{1 \leq i, j \leq N} \xi_{i} A_{i j} \xi_{j}^{\prime}
$$

This last condition (7) will guarantee the linear unstability of the zero state and the existence and positivity of the spreading speeds in slowly oscillating media, as defined below (see Proposition 2.1). Notice in particular that, if $b=0$ in $\mathbb{R}^{N}$, then condition (7) is fulfilled if and only if $\max _{\mathbb{R}^{N}} c>0$.

For any $L>0$, we define the $L$-periodic rescaled coefficients $a_{L}, b_{L}$ and $c_{L}$ by:

$$
a_{L}(x)=a\left(\frac{x}{L}\right), \quad b_{L}(x)=b\left(\frac{x}{L}\right), \quad c_{L}(x)=c\left(\frac{x}{L}\right) \quad \text { for all } x \in \mathbb{R}^{N} .
$$


Then, because of (5-6), there exists, for each $\lambda \in \mathbb{R}^{N}$ and $L>0$, a periodic principal eigenvalue $k_{L}(\lambda)$ which is uniquely defined by the existence of a function $\phi_{L, \lambda} \in \bigcap_{1 \leq p<+\infty} W^{2, p}\left(\mathbb{R}^{N}\right)$ such that

$$
\left\{\begin{array}{l}
\operatorname{div}\left(a_{L} \nabla \phi_{L, \lambda}\right)-2 \lambda a_{L} \nabla \phi_{L, \lambda}+b_{L} \cdot \nabla \phi_{L, \lambda} \\
\quad+\left(\lambda a_{L} \lambda-\operatorname{div}\left(a_{L} \lambda\right)-b_{L} \cdot \lambda+c_{L}\right) \phi_{L, \lambda}=k_{L}(\lambda) \phi_{L, \lambda} \text { in } \mathbb{R}^{N}, \\
\phi_{L, \lambda} \text { is } L \text {-periodic, } \\
\phi_{L, \lambda}>0 \text { in } \mathbb{R}^{N},
\end{array}\right.
$$

see e.g. [39]. The maps $\lambda \mapsto k_{L}(\lambda)$ are known to be convex (see [4]).

For any $L>0$ and any unit direction $e \in \mathbb{S}^{N-1}$, we then define

$$
w_{L}^{*}(e):=\inf _{\lambda \in \mathbb{R}^{N}, \lambda \cdot e>0} \frac{k_{L}(\lambda)}{\lambda \cdot e} .
$$

In this general setting, the quantities $w_{L}^{*}(e)$ defined in formula (9) are a priori not directly associated with an equation of the type (1) and with some spreading speeds in the sense of (4). However, the numbers $w_{L}^{*}(e)$ can really be viewed as spreading speeds under some reasonable assumptions that we give now. Namely, in addition to (5-6), assume here that

$$
f: \mathbb{R}^{N} \times[0,+\infty) \rightarrow \mathbb{R}, \quad(x, s) \mapsto f(x, s)
$$

is continuous, 1 -periodic in $x$, that $c:=\frac{\partial f}{\partial s}(\cdot, 0)$ is continuous and that $f$ satisfies $(2)$ and (3). If $k_{L}(\lambda)>0$ for all $\lambda \in \mathbb{R}^{N}$, then the following holds: for any nonzero, nonnegative and compactly supported function $u_{0} \in L^{\infty}\left(\mathbb{R}^{N}\right)$, the solution $u$ of (1) with initial datum $u_{0}$ propagates in the sense of (4) with the spreading speed $w_{L}^{*}(e)>0$ given by formula (9), in any direction $e \in \mathbb{S}^{N-1}$, see $[6,41]$. This is why we always say in this paper that the quantities $w_{L}^{*}(e)$ represent the spreading speeds of the solutions of some reaction-diffusionadvection equations of the type (1).

The following proposition ensures that the aforementioned positivity condition on the real numbers $k_{L}(\lambda)$ and the positivity of the spreading speeds $w_{L}^{*}(e)$ are fulfilled, at least when $L$ is large enough.

Proposition 2.1 Under the above assumptions (5-7), there is $L_{0}>0$ such that, for all $L \geq L_{0}$, there holds $\min _{\lambda \in \mathbb{R}^{N}} k_{L}(\lambda)>0$ and, for any direction $e \in \mathbb{S}^{N-1}$, the infimum in $(9)$ is reached and positive.

\subsection{Convergence of the speed when $L \rightarrow+\infty$ and computation of the limit in dimension 1}

When $b$ is divergence-free, the functions $L \mapsto k_{L}(\lambda)$ are nondecreasing (see [33]), whence the limit $w_{\infty}^{*}(e):=\lim _{L \rightarrow \infty} w_{L}^{*}(e)$ is well-defined in $\overline{\mathbb{R}}$ for any direction $e$. In the general case considered here, the main goal of the present work is twofold: firstly, proving the

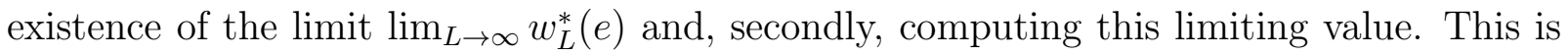
the purpose of the following theorems. 
Theorem 2.2 Under assumptions (5-7), for any unit vector $e \in \mathbb{S}^{N-1}$, the limit $w_{\infty}^{*}(e)=\lim _{L \rightarrow+\infty} w_{L}^{*}(e)$ exists and is a positive real number. Furthermore,

$$
w_{\infty}^{*}(e)=\min _{\lambda \in \mathbb{R}^{N}, \lambda \cdot e>0} \frac{k_{\infty}(\lambda)}{\lambda \cdot e},
$$

where the function $k_{\infty}: \mathbb{R}^{N} \rightarrow \mathbb{R}$ is defined in Proposition 3.1 below.

In dimension $N=1$, the quantities $k_{\infty}(\lambda)$ can be given in terms of explicit integrals involving the underlying coefficients.

Theorem 2.3 Under assumptions (5-7), in dimension $N=1$, there holds

$$
w_{\infty}^{*}( \pm 1)=\min _{\lambda \geq j_{ \pm}(M), \lambda>0} \frac{j_{ \pm}^{-1}(\lambda)}{\lambda}
$$

where the functions $j_{ \pm}:[M,+\infty) \rightarrow\left[j_{ \pm}(M),+\infty\right)$ are defined by

$$
j_{ \pm}(k)=\int_{0}^{1} \sqrt{\frac{k-c(x)}{a(x)}+\frac{b^{2}(x)}{4 a^{2}(x)}} d x \pm \int_{0}^{1} \frac{b(x)}{2 a(x)} d x
$$

for all $k \geq M^{1}$

In particular, in dimension $N=1$, when $a=1$ and $b=0$, we get

$$
j_{ \pm}(k)=\int_{0}^{1} \sqrt{k-c(x)} d x
$$

and (11) corresponds to the formula conjectured by Hamel, Fayard and Roques in [21]. Note that, in that case, and when $c$ is a step function, it has been shown in [21] that (11) leads to an even more explicit formula. More generally speaking, it is noteworthy that formula (11) allows for easy numerical computations of the (equal) limiting spreading speeds $w_{\infty}^{*}( \pm 1)$.

We conjecture that Theorem 2.2 can be extended to the case of space-time periodic coefficients. Assume that $a=a(t, x), b=b(t, x)$ and $f=f(t, x, s)$ are 1-periodic with respect to $t$ and $x$, and consider the equation

$$
\frac{\partial u}{\partial t}=\operatorname{div}\left(a_{L}(t, x) \nabla u\right)+b_{L}(t, x) \cdot \nabla u+f_{L}(t, x, u), \quad t>0, \quad x=\left(x_{1}, \ldots, x_{N}\right) \in \mathbb{R}^{N}
$$

with slow variations in space and in time

$$
a_{L}(t, x)=a\left(\frac{t}{L}, \frac{x}{L}\right), \quad b_{L}(t, x)=b\left(\frac{t}{L}, \frac{x}{L}\right), \quad f_{L}(t, x, s)=f\left(\frac{t}{L}, \frac{x}{L}, s\right) .
$$

\footnotetext{
${ }^{1}$ Notice that the values $j_{ \pm}(M)$ may not be positive. Indeed, for instance, if the functions $a, b$ and $c$ are all constants and fulfill (6) and (7), then $j_{ \pm}(M)= \pm b /(2 a)$ and $j_{ \pm}(M)$ are then of the sign of $\pm b$.
} 
The existence of pulsating traveling fronts for equation (13) has been proved by Nolen, Rudd and Xin [34] when $f$ and $a$ do not depend on $(t, x), f(0)=f(1)=0, f(s)>0$ if $s \in(0,1)$ and $b$ is incompressible. This result has been extended by the second author [31] when $a$ and $f$ are space-time periodic and $b$ is a general space-time periodic vector field. When $f$ satisfies the KPP assumption of the type (3) with time-dependence, the first two authors, together with Berestycki, proved in [6] the existence of a spreading speed $w_{L}^{*}(e)$ associated with (13) so that (4) holds. A formula like (9) still holds, except that $k_{L}(\lambda)$ is not associated with an elliptic operator anymore but with a parabolic operator with time periodicity conditions (see [6]). Then, it should be possible to extend the result of Theorem 2.2 and Proposition 3.1 below, but now $k_{\infty}(\lambda)$ is uniquely defined by the existence of a space-time periodic viscosity solution $Z \in \mathcal{C}^{0}\left(\mathbb{R} \times \mathbb{R}^{N}\right)$ of

$$
-\partial_{t} Z+(\nabla Z-\lambda) a(\nabla Z-\lambda)+b \cdot(\nabla Z-\lambda)+c=k_{\infty}(\lambda) .
$$

The dependence of $k_{L}(\lambda)$ and $k_{\infty}(\lambda)$ with respect to the coefficients $(a, b, c)$ is a topical subject (see [32, 35] for a few results concerning $k_{L}(\lambda)$ ). Nolen and Xin conjectured in [35] that temporal variations should slow down the propagation. This is still an open question. A partial answer is given by our result together with numerical simulations carried out by Khouider, Bourlioux and Majda [25], who observed that when $a=I_{N}, c$ does not depend on $(t, x)$ and $b\left(t, x_{1}, x_{2}, \ldots, x_{N}\right)=\widetilde{b}\left(\omega t, x_{2}, \ldots, x_{N}\right) e_{1}$ is a shear flow in the direction $e_{1}=(1,0, \ldots, 0)$ with temporal frequency $\omega$, then the spreading speed $w_{\infty}^{*}\left(e_{1}\right)$ is decreasing with respect to $\omega$.

\subsection{The case of homogeneous reaction terms with no advection}

In another particular case, we immediately get a simpler formula for $w_{\infty}^{*}( \pm 1)$ in dimension $N=1$.

Corollary 2.4 Under the conditions of Theorem 2.3, if $b=0$ and $c=c_{0}>0$ is constant, then

$$
w_{\infty}^{*}( \pm 1)=2<\sqrt{a}>_{H} \sqrt{c_{0}},
$$

where

$$
<\sqrt{a}>_{H}=\left(\int_{0}^{1} \frac{d x}{\sqrt{a(x)}}\right)^{-1}
$$

denotes the harmonic mean of $\sqrt{a}$.

Notice that, under the conditions of Corollary 2.4, it is also known that

$$
w_{L}^{*}( \pm 1) \rightarrow 2 \sqrt{<a>_{H}} \sqrt{c_{0}} \text { as } L \rightarrow 0^{+}
$$

see [12]. More generally speaking, if the arithmetic mean $\langle c\rangle_{A}=\int_{0}^{1} c(x) d x$ is positive, then

$$
\lim _{L \rightarrow 0^{+}} w_{L}^{*}( \pm 1)=2 \sqrt{\left\langle a>_{H}\right.} \sqrt{\left\langle c>_{A}\right.}
$$



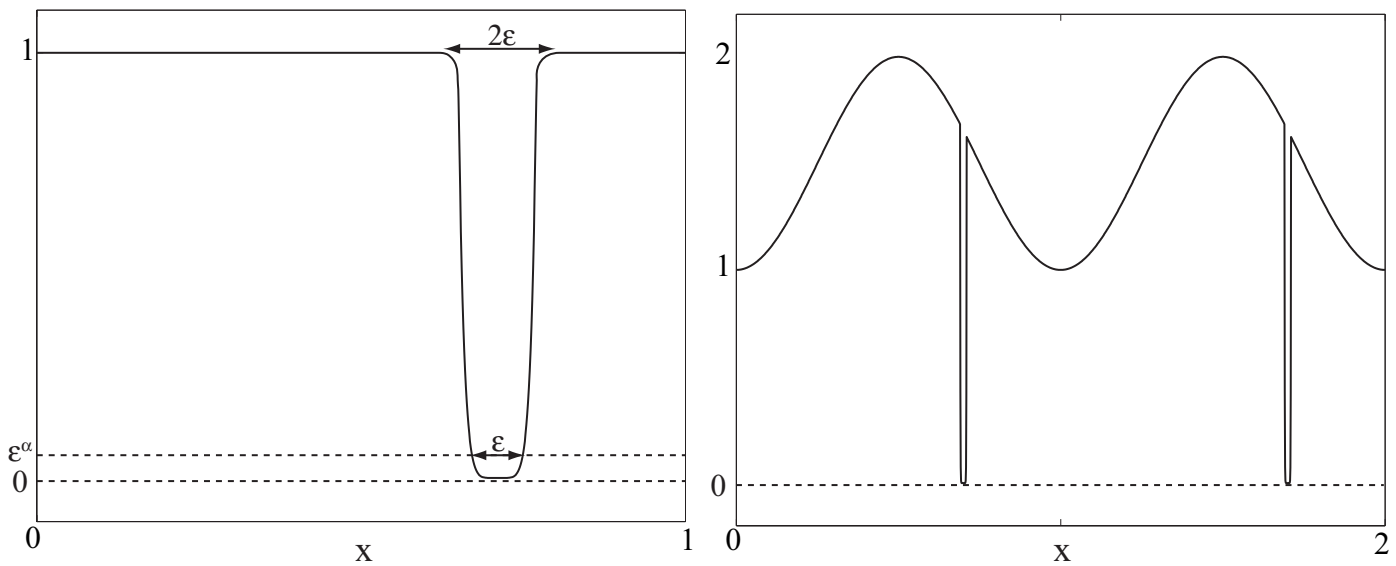

Figure 1: (left) Schematic plot of a perturbation function $\rho_{\varepsilon}$; (right) A perturbed function $x \mapsto \rho_{\varepsilon}(x)\left(1+\sin ^{2}(\pi x)\right)$, with $\varepsilon=10^{-2}$ and $\alpha=3$ in the definition of $\rho_{\varepsilon}$.

Observe now that, because of Cauchy-Schwarz inequality, the right-hand side of (15) is (strictly) larger than the right-hand side of (16) as soon as the diffusion rate $a$ is not constant (this is coherent with the fact that the spreading speeds $w_{L}^{*}( \pm 1)$ are non-decreasing with respect to $L$ in this case, see [33]). Furthermore, the ratio $\langle\sqrt{a}\rangle_{H} / \sqrt{\langle a\rangle_{H}} \geq 1$ then measures the relative increase of the spreading speeds from a completely homogenized medium to the infinitely slowly oscillating one.

\subsection{Respective effects of perturbations of the reaction term and the diffusion term on the speed}

The above results have further interesting consequences regarding the respective effects of localized perturbations of the reaction and diffusion terms on the spreading speeds. To make the dependence more transparent, let us use the notations

$$
k_{L, a, b, c}(\lambda), \quad w_{L, a, b, c}^{*}(e) \text { and } w_{\infty, a, b, c}^{*}(e)
$$

for the quantities defined in (8), (9) and (10).

For the sake of simplicity of the presentation, assume that the dimension is $N=1$ and that $b=0$. Assume that $a$ and $c$ fulfill (5-6) and $c$ has a positive average (whence $\min _{\lambda \in \mathbb{R}} k_{L, a, 0, c}(\lambda)>0$ and $w_{L, a, 0, c}^{*}( \pm 1)>0$ for all $\left.L>0\right)$.

Then, consider a real number $\alpha>2$ and a family of smooth 1-periodic positive functions $\left(\rho_{\varepsilon}\right)_{0<\varepsilon<1 / 2}$ such that, on each period,

$$
\begin{cases}0<\rho_{\varepsilon} \leq 1, & \\ 0<\rho_{\varepsilon} \leq \varepsilon^{\alpha} & \text { in an interval of length } \varepsilon, \\ \rho_{\varepsilon}=1 & \text { in an interval of length } 1-2 \varepsilon\end{cases}
$$

see Fig. 1. 
Consider now the case when the diffusion coefficient is multiplied by the perturbation $\rho_{\varepsilon}$, that is when $a$ is replaced with $\rho_{\varepsilon} \times a$ : it follows from the maximum principle and Corollary 2.4 together with the monotonicity of the maps $L \mapsto w_{L, \rho_{\varepsilon} a, 0, c}^{*}( \pm 1)$ (because $b=0$ ), that the upper bound holds

$$
0<w_{L, \rho_{\varepsilon} a, 0, c}^{*}( \pm 1) \leq w_{L, \rho_{\varepsilon} a, 0, M_{c}}^{*}( \pm 1) \leq w_{\infty, \rho_{\varepsilon} a, 0, M_{c}}^{*}( \pm 1) \leq 2 \varepsilon^{\alpha / 2-1} \sqrt{M_{a}} \sqrt{M_{c}},
$$

where $M_{a}=\max _{\mathbb{R}} a$ and $M_{c}=\max _{\mathbb{R}} c$. As a consequence,

$$
\forall L>0, \quad w_{L, \rho_{\varepsilon} a, 0, c}^{*}( \pm 1) \rightarrow 0 \text { as } \varepsilon \rightarrow 0 .
$$

On the other hand, when the growth rate $c$ is multiplied by the perturbation $\rho_{\varepsilon}$ (that is when the nonlinearity $f_{L}(x, u)$ is replaced with $\rho_{\varepsilon}(x / L) \times f_{L}(x, u)$ in equation (1), where $b_{L}=0, f$ satisfies $(2)$ and $(3)$ and $c$ denotes $\left.c=\frac{\partial f}{\partial s}(\cdot, 0)\right)$, then the associated spreading speeds satisfy (see $[12,33]$ ), for all $L>0$,

$$
w_{L, a, 0, \rho_{\varepsilon} c}^{*}( \pm 1) \geq \lim _{L^{\prime} \rightarrow 0} w_{L^{\prime}, a, 0, \rho_{\varepsilon} c}^{*}( \pm 1)=2 \sqrt{<a>_{H}} \sqrt{\left\langle\rho_{\varepsilon} c>_{A}\right.} .
$$

Thus, it follows from (18) that

$$
\forall L>0, \quad \liminf _{\varepsilon \rightarrow 0} w_{L, a, 0, \rho_{\varepsilon} c}^{*}( \pm 1) \geq 2 \sqrt{\langle a\rangle_{H}} \sqrt{\langle c\rangle_{A}}>0 .
$$

In short, formulas (19) and (20) show that small perturbations of the diffusion coefficient, in the sense above, can dramatically reduce the spreading speeds whereas similar perturbations of the reaction term have a limited effect.

\subsection{Large amplitude of the reaction and advection terms}

Another consequence of Theorem 2.2 is concerned with the determination of the asymptotic dependence with respect to the drift and reaction terms in the large amplitude case.

Corollary 2.5 Under the assumptions of Theorem 2.2 and notations (17), there holds, for any $L>0$ and for any unit vector $e \in \mathbb{S}^{N-1}$,

$$
\lim _{B \rightarrow+\infty} \frac{w_{L, a, \sqrt{B} b, B c}^{*}(e)}{\sqrt{B}}=\lim _{L \rightarrow+\infty} w_{L, a, b, c}^{*}(e)=w_{\infty, a, b, c}^{*}(e) .
$$

In particular, consider the one-dimensional symmetric equation

$$
\frac{\partial u}{\partial t}=\frac{\partial}{\partial x}\left(a_{L}(x) \frac{\partial u}{\partial x}\right)+B f_{L}(x, u)
$$

where $f: \mathbb{R} \times[0,+\infty) \times \mathbb{R}$ is continuous, 1-periodic in $x$ and satisfies (2) and (3). Assume that $c=\frac{\partial f}{\partial s}(\cdot, 0)$ is continuous. If $\min _{\mathbb{R}} a>0$ and $M=\max _{\mathbb{R}} c>0$, then Corollary 2.5 gives an equivalent asymptotics for the spreading speeds $w_{L, a, 0, B c}^{*}( \pm 1)$ of the solutions 
of $(21)$ in the limit of large amplitudes $B$ of the reaction term. Namely, for any $L>0$, we have

$$
w_{L, a, 0, B c}^{*}( \pm 1) \sim\left(\min _{\lambda \geq j(M)} \frac{j^{-1}(\lambda)}{\lambda}\right) \times \sqrt{B} \text { as } B \rightarrow+\infty,
$$

where $j(k)=\int_{0}^{1} \sqrt{\frac{k-c(x)}{a(x)}} d x$ for all $k \geq M$ (notice that the coefficient $\min _{\lambda \geq j(M)} j^{-1}(\lambda) / \lambda$ is necessarily positive). This result improves Theorem 1.3 of [9] in the one-dimensional case.

\section{Convergence of the periodic principal eigenvalues as $L \rightarrow+\infty$}

The aim of this section is to prove the existence of the limit of the principal eigenvalues $k_{L}(\lambda)$, defined in (8), as $L \rightarrow+\infty$ and to express it explicitly in dimension 1 . We shall especially use the Hopf-Cole transformation of the eigenfunctions and the stability theorem for Hamilton-Jacobi equations with small viscosity.

Proposition 3.1 Under assumptions (5-6), for all $\lambda \in \mathbb{R}^{N}$, the limit

$$
k_{\infty}(\lambda)=\lim _{L \rightarrow+\infty} k_{L}(\lambda)
$$

exists and it is the unique real number $k_{\infty}(\lambda)$ such that there exists a continuous 1-periodic viscosity solution $Z$ of the cell problem

$$
(\nabla Z-\lambda) a(\nabla Z-\lambda)+b \cdot(\nabla Z-\lambda)+c=k_{\infty}(\lambda) .
$$

Furthermore,

$$
k_{\infty}(\lambda) \geq M=\max _{x \in \mathbb{R}^{N}}\left(c(x)-\frac{b(x) a(x)^{-1} b(x)}{4}\right)
$$

for all $\lambda \in \mathbb{R}^{N}$. Lastly, the function $\lambda \mapsto k_{\infty}(\lambda)$ is convex and the convergence $k_{L}(\lambda) \rightarrow k_{\infty}(\lambda)$ as $L \rightarrow+\infty$ is locally uniform with respect to $\lambda \in \mathbb{R}^{N}$.

Proof. Let $\lambda$ be any fixed vector in $\mathbb{R}^{N}$ and denote by $|\lambda|$ its euclidean norm. First, it follows from (6) and (8) that

$$
\begin{aligned}
& \nu|\lambda|^{2}-L^{-1}\|\operatorname{div}(a \lambda)\|_{\infty}-\|b\|_{\infty}|\lambda|-\|c\|_{\infty} \\
& \quad \leq k_{L}(\lambda) \leq \max _{\mathbb{R}^{N}}(\lambda a \lambda)+L^{-1}\|\operatorname{div}(a \lambda)\|_{\infty}+\|b\|_{\infty}|\lambda|+\|c\|_{\infty}
\end{aligned}
$$

for all $L>0$. Therefore, the family $\left(k_{L}(\lambda)\right)_{L \geq 1}$ is bounded. There exist then a real number $k_{\infty}(\lambda)$ and a sequence $\left(L_{n}\right)_{n \in \mathbb{N}} \rightarrow+\infty$ such that

$$
k_{L_{n}}(\lambda) \rightarrow k_{\infty}(\lambda) \text { as } n \rightarrow+\infty
$$


For each $n \in \mathbb{N}$, let $\phi_{L_{n}, \lambda} \in \bigcap_{1 \leq p<+\infty} W^{2, p}\left(\mathbb{R}^{N}\right)$ be the principal $L_{n}$-periodic eigenvalue of (8) with $L=L_{n}$. Up to normalization, one can assume that $\phi_{L_{n}, \lambda}(0)=1$. The function $\phi_{L_{n}, \lambda}$ is positive in $\mathbb{R}^{N}$. Define

$$
Z_{n}(x)=\frac{\ln \phi_{L_{n}, \lambda}\left(L_{n} x\right)}{L_{n}}
$$

for all $x \in \mathbb{R}^{N}$. The 1 -periodic function $Z_{n} \in \bigcap_{1 \leq p<+\infty} W^{2, p}\left(\mathbb{R}^{N}\right)$ satisfies $Z_{n}(0)=0$ and $L_{n}^{-1} \operatorname{div}\left(a \nabla Z_{n}\right)+\nabla Z_{n} a \nabla Z_{n}-2 \lambda a \nabla Z_{n}+b \cdot \nabla Z_{n}+\lambda a \lambda-L_{n}^{-1} \operatorname{div}(a \lambda)-b \cdot \lambda+c=k_{L_{n}}(\lambda)$.

On the other hand, since the functions $\phi_{L_{n}, \lambda}$ satisfy (8) with coefficients which are uniformly bounded (since $L_{n} \rightarrow+\infty$ ) and fulfill (6), it follows from the standard interior elliptic estimates that there exists a constant $C_{1}>0$ such that

$$
\left|\nabla \phi_{L_{n}, \lambda}(x)\right| \leq C_{1} \times \max _{|y-x| \leq 1} \phi_{L_{n}, \lambda}(y)
$$

for all $n \in \mathbb{N}$ and $x \in \mathbb{R}^{N}$. But, since the functions $\phi_{L_{n}, \lambda}$ are all positive, the Harnack inequality yields the existence of a constant $C_{2}>0$ such that

$$
\max _{|y-x| \leq 1} \phi_{L_{n}, \lambda}(y) \leq C_{2} \times \min _{|y-x| \leq 1} \phi_{L_{n}, \lambda}(y) \leq C_{2} \times \phi_{L_{n}, \lambda}(x)
$$

for all $n \in \mathbb{N}$ and $x \in \mathbb{R}^{N}$. Therefore, the fields $\nabla \phi_{L_{n}, \lambda} / \phi_{L_{n}, \lambda}$ are bounded in $L^{\infty}\left(\mathbb{R}^{N}\right)$ uniformly with respect to $n \in \mathbb{N}$. In other words, the sequence $\left(\left\|\nabla Z_{n}\right\|_{\infty}\right)_{n \in \mathbb{N}}$ is bounded. As $Z_{n}$ is 1-periodic and $Z_{n}(0)=0$ for all $n \in \mathbb{N}$, the sequence $\left(Z_{n}\right)_{n \in \mathbb{N}}$ is then bounded in $W^{1, \infty}\left(\mathbb{R}^{N}\right)$. Ascoli's theorem implies that, up to extraction of a subsequence, the sequence $\left(Z_{n}\right)_{n \in \mathbb{N}}$ converges uniformly in $\mathbb{R}^{N}$ to a Lipschitz-continuous 1-periodic function $Z$. The stability theorem for Hamilton-Jacobi equations (see $[2,3]$ ) gives that $Z$ is a viscosity solution of $(22)$.

The uniqueness of the real number $k_{\infty}(\lambda)$ such that (22) admits a periodic viscosity solution follows from a classical theorem by Lions, Papanicolaou and Varadhan (unpublished manuscript, see also [13]). As a consequence, the whole family $\left(k_{L}(\lambda)\right)_{L>0}$ converges to $k_{\infty}(\lambda)$ as $L \rightarrow+\infty$.

By construction, since the function $Z$ is Lipschitz-continuous, it follows from Rademacher's theorem that equation $(22)$ is satisfied almost everywhere in $\mathbb{R}^{N}$. Therefore, (23) holds immediately, since the fields $a, b$ and $c$ are continuous in $\mathbb{R}^{N}$. Lastly, since all functions $\lambda \mapsto k_{L}(\lambda)$ were convex, the function $\lambda \mapsto k_{\infty}(\lambda)$ is convex too, whence continuous. Because of $(24)$ and the convexity of $k_{L}$, the slopes

$$
\lambda \mapsto \lim _{t \rightarrow 0^{+}} \frac{k_{L}(\lambda+t \xi)-k_{L}(\lambda)}{t}
$$

are locally bounded, uniformly with respect to $L \geq 1$ and $\xi \in \mathbb{S}^{N-1}$. As a consequence, the limit $k_{\infty}(\lambda)=\lim _{L \rightarrow+\infty} k_{L}(\lambda)$ is locally uniform. The proof of Proposition 3.1 is thereby 
complete.

An immediate consequence of Proposition 3.1 is the

Proof of Proposition 2.1. In addition to (5-6), assume here that (7) holds, that is $M>0$. It follows from (24) that there exists $r>0$ such that

$$
\forall L \geq 1, \forall|\lambda| \geq r, \quad k_{L}(\lambda) \geq \nu \frac{|\lambda|^{2}}{2} .
$$

On the other hand, since the convergence $\lim _{L \rightarrow+\infty} k_{L}(\lambda)=k_{\infty}(\lambda) \geq M>0$ is locally uniform with respect to $\lambda \in \mathbb{R}^{N}$, there is $L_{0} \geq 1$ such that

$$
\forall L \geq L_{0}, \forall|\lambda| \leq r, \quad k_{L}(\lambda) \geq \frac{M}{2}
$$

In particular, for any $L \geq L_{0}$, the infimum over $\mathbb{R}^{N}$ of the continuous function $k_{L}: \lambda \mapsto k_{L}(\lambda)$ is reached and positive.

Let now $e$ be any unit vector of $\mathbb{S}^{N-1}$. It follows straightforwardly from (25) and (26) that, for any $L \geq L_{0}$, the quantity $w_{L}^{*}(e)$ defined in (9) is a minimum and is positive.

Lastly, in dimension $N=1$, we can now give an explicit expression of the limit $k_{\infty}(\lambda)$ for each $\lambda \in \mathbb{R}$, in terms of some integral quantities.

Proposition 3.2 Under the assumptions and notations of Proposition 3.1, let

$$
j_{ \pm}:[M,+\infty) \rightarrow\left[j_{ \pm}(M),+\infty\right)
$$

be the functions given as in (12). In dimension 1 , there holds, for all $\lambda \in \mathbb{R}$,

$$
k_{\infty}(\lambda)= \begin{cases}\text { the unique } k \geq M \text { such that } \lambda=-j_{-}(k) & \text { if } \lambda \leq-j_{-}(M), \\ M & \text { if }-j_{-}(M)<\lambda<j_{+}(M), \\ \text { the unique } k \geq M \text { such that } \lambda=j_{+}(k) & \text { if } \lambda \geq j_{+}(M) .\end{cases}
$$

Proof. First, let $\lambda \geq j_{+}(M)$. Since the function $j_{+}$is one-to-one and onto from $[M,+\infty)$ to $\left[j_{+}(M),+\infty\right)$, there exists a unique real number $k \geq M$ such that:

$$
\lambda=j_{+}(k)=\int_{0}^{1} \sqrt{\frac{k-c(x)}{a(x)}+\frac{b^{2}(x)}{4 a^{2}(x)}} d x+\int_{0}^{1} \frac{b(x)}{2 a(x)} d x .
$$

Define, for all $x \in \mathbb{R}$,

$$
Z(x)=\lambda x-\int_{0}^{x} \sqrt{\frac{k-c(y)}{a(y)}+\frac{b^{2}(y)}{4 a^{2}(y)}} d y-\int_{0}^{x} \frac{b(y)}{2 a(y)} d y .
$$


As $k$ satisfies $(28)$, the function $Z$ is 1 -periodic and belongs to $W^{1, \infty}(\mathbb{R})$. Moreover, one has

$$
a\left(Z^{\prime}-\lambda+\frac{b}{2 a}\right)^{2}=k-c+\frac{b^{2}}{4 a}
$$

almost everywhere in $\mathbb{R}$ and finally $Z$ satisfies $(22)$. Thus the uniqueness of $k_{\infty}(\lambda)$ yields $k=k_{\infty}(\lambda)$.

Similarly, one can check that, if $\lambda \leq-j_{-}(M), k_{\infty}(\lambda)$ is the unique real number $k \in[M,+\infty)$ such that $\lambda=-j_{-}(k)$.

In particular, there holds

$$
k_{\infty}\left(-j_{-}(M)\right)=k_{\infty}\left(j_{+}(M)\right)=M
$$

But since the function $k_{\infty}$ is convex and bounded from below by $M$ (from Proposition 3.1), one concludes that $k_{\infty}(\lambda)=M$ if $\lambda \in\left[-j_{-}(M), j_{+}(M)\right]$, which completes the proof of Proposition 3.2.

\section{Proof of Theorems 2.2 and 2.3 and their corollaries}

This section is devoted to the proof of the main results announced in Section 2, making use of the propositions and estimates of the previous section. We start with the proof of Theorem 2.2 for the general higher-dimensional case $N \geq 1$.

Proof of Theorem 2.2. In addition to (5-6), we assume that (7) holds, that is $M>0$. Let $e \in \mathbb{S}^{N-1}$ be any unit vector of $\mathbb{R}^{N}$. We recall that, for each $L>0$, the spreading speed $w_{L}^{*}(e)$ is defined as

$$
w_{L}^{*}(e)=\inf _{\lambda \in \mathbb{R}^{N}, \lambda \cdot e>0} \frac{k_{L}(\lambda)}{\lambda \cdot e} .
$$

From Proposition 2.1, there exists $L_{0}>0$ such that $k_{L}(0)>0$ and the infimum in the above formula is reached and positive. Let $\left(L_{n}\right)_{n \in \mathbb{N}}$ be any sequence such that $L_{n} \geq L_{0}$ for all $n \in \mathbb{N}$, and $L_{n} \rightarrow+\infty$ as $n \rightarrow+\infty$. For each $n \in \mathbb{N}$, let then $\lambda_{n} \in \mathbb{R}^{N}$ be such that $\lambda_{n} \cdot e>0$ and

$$
w_{L_{n}}^{*}(e)=\frac{k_{L_{n}}\left(\lambda_{n}\right)}{\lambda_{n} \cdot e}>0 .
$$

Taking $\lambda=\kappa e$ in (24) for any $\kappa>0$, and using the definition (29), we get

$$
w_{L}^{*}(e) \leq 2 \sqrt{\|c\|_{\infty} \max _{\mathbb{R}^{N}}(e a e)}+L^{-1}\|\operatorname{div}(a e)\|_{\infty}+\|b\|_{\infty}
$$

for all $L>0$. Therefore, the sequence $\left(w_{L_{n}}(e)\right)_{n \in \mathbb{N}}$ is bounded and then converges, up to extraction of a sequence, to a nonnegative real number $w_{\infty}^{*}(e)$.

Next, taking $\lambda=\lambda_{n}$ in (24), one gets

$$
\nu\left|\lambda_{n}\right|^{2}-L_{n}^{-1}\left\|\operatorname{div}\left(a \lambda_{n}\right)\right\|_{\infty}-\left|\lambda_{n}\right|\|b\|_{\infty}-\|c\|_{\infty} \leq k_{L_{n}}\left(\lambda_{n}\right)=w_{L_{n}}^{*}(e) \times\left(\lambda_{n} \cdot e\right) \leq w_{L_{n}}^{*}(e) \times\left|\lambda_{n}\right|
$$


for all $n \in \mathbb{N}$. Therefore, the sequence $\left(\lambda_{n}\right)_{n \in \mathbb{N}}$ is bounded too and then converges, up to extraction of another sequence, to a vector $\lambda_{\infty} \in \mathbb{R}^{N}$ such that $\lambda_{\infty} \cdot e \geq 0$.

On the other hand, since, from Proposition 3.1, the convergence of the family of functions $\left(k_{L}\right)_{L>0}$ to the function $k_{\infty}$ as $L \rightarrow+\infty$ is locally uniform, it follows that

$$
k_{\infty}\left(\lambda_{\infty}\right)=w_{\infty}^{*}(e) \times\left(\lambda_{\infty} \cdot e\right) .
$$

Since $k_{\infty}(\lambda) \geq M>0$ for all $\lambda \in \mathbb{R}^{N}$ from Proposition 3.1 and assumption (7), one concludes that $w_{\infty}^{*}(e)$ and $\lambda_{\infty} \cdot e$ are both positive, and

$$
w_{\infty}^{*}(e)=\frac{k_{\infty}\left(\lambda_{\infty}\right)}{\lambda_{\infty} \cdot e}
$$

For all $\lambda \in \mathbb{R}^{N}$ such that $\lambda \cdot e>0$, one has

$$
w_{L_{n}}^{*}(e) \leq \frac{k_{L_{n}}(\lambda)}{\lambda \cdot e}
$$

for all $n \in \mathbb{N}$, whence

$$
w_{\infty}^{*}(e) \leq \frac{k_{\infty}(\lambda)}{\lambda \cdot e} .
$$

Finally, the function $\lambda \mapsto k_{\infty}(\lambda) /(\lambda \cdot e)$ reaches its minimum at $\lambda_{\infty}$ over the set of $\lambda \in \mathbb{R}^{N}$ such that $\lambda \cdot e>0$, and

$$
w_{\infty}^{*}(e)=\min _{\lambda \in \mathbb{R}^{N}, \lambda \cdot e>0} \frac{k_{\infty}(\lambda)}{\lambda \cdot e} .
$$

In particular, from the uniqueness of the limit, one concludes that the whole family $\left(w_{L}^{*}(e)\right)_{L>0}$ converges to $w_{\infty}^{*}(e)$ as $L \rightarrow+\infty$. The proof of Theorem 2.2 is thereby complete.

The proof of Theorem 2.3 in dimension $N=1$ is based on Theorem 2.2 and on the expression of $k_{\infty}(\lambda)$ given in Proposition 3.2.

Proof of Theorem 2.3. We only prove the formula for $w_{\infty}^{*}(+1)$, the case of $w_{\infty}^{*}(-1)$ could be treated similarly. Two cases have to be considered, according to the sign of $j_{+}(M)$. Let us first assume that $j_{+}(M)>0$. From Proposition 3.1, there holds

$$
\frac{k_{\infty}(\lambda)}{\lambda} \geq \frac{M}{\lambda} \text { for all } \lambda \in\left(0, j_{+}(M)\right)
$$

while

$$
\frac{k_{\infty}(\lambda)}{\lambda}=\frac{j^{-1}(\lambda)}{\lambda} \text { for all } \lambda \geq j_{+}(M)
$$

from Proposition 3.2. It follows then from (30) that the function $\lambda \mapsto j_{+}^{-1}(\lambda) / \lambda$ has a minimum on the interval $\left[j_{+}(M),+\infty\right)$, and that

$$
w_{\infty}^{*}(+1)=\min _{\lambda \geq j_{+}(M)} \frac{j_{+}^{-1}(\lambda)}{\lambda},
$$


which is the desired conclusion (11) in the case $j_{+}(M)>0$.

Lastly, if $j_{+}(M) \leq 0$, then $k_{\infty}(\lambda)=j_{+}^{-1}(\lambda)$ for all $\lambda>0$ from Proposition 3.2, and (11) follows immediately from (30). That completes the proof of Theorem 2.3.

The proofs of Corollaries 2.4 and 2.5 then follow easily from Theorems 2.3 and 2.2 respectively.

Proof of Corollary 2.4. In dimension $N=1$, when $b=0$ and $c_{0}>0$ is constant, then $M=c_{0}, j_{ \pm}(M)=0$ and

$$
j_{ \pm}(k)=\sqrt{k-c_{0}} \int_{0}^{1} \frac{d x}{\sqrt{a(x)}}
$$

for all $k \geq M$. Therefore, $j_{ \pm}^{-1}(\lambda)=<\sqrt{a}>_{H}^{2} \lambda^{2}+c_{0}$ for all $\lambda \geq 0$ and the conclusion (15) follows from Theorem 2.3.

Lastly, Corollary 2.5 is just a consequence of Theorem 2.2 and of a rescaling argument.

Proof of Corollary 2.5. For each $L>0$ and $\lambda \in \mathbb{R}^{N}$, we call $k_{L, a, b, c}(\lambda)$ and $\phi_{L, a, b, c, \lambda}$ the (unique) principal eigenvalue and eigenfunction of (8) and we also use the notation $w_{L, a, b, c}^{*}(e)$ for the quantities defined in (9). Now, for any $L>0, B>0$ and $\lambda \in \mathbb{R}^{N}$, the function $\psi$ defined by

$$
\psi(x)=\phi_{\sqrt{B} L, a, b, c, \lambda}(\sqrt{B} x)
$$

is positive, $L$-periodic and it satisfies

$$
\begin{aligned}
\operatorname{div}\left(a_{L} \nabla \psi\right)-2 \sqrt{B} & \lambda a_{L} \nabla \psi+\sqrt{B} b_{L} \cdot \nabla \psi \\
& +\left(B \lambda a_{L} \lambda-\sqrt{B} \operatorname{div}\left(a_{L} \lambda\right)-B b_{L} \cdot \lambda+B c_{L}\right) \psi=B k_{\sqrt{B}{ }_{L, a, b, c}}(\lambda) \psi
\end{aligned}
$$

in $\mathbb{R}^{N}$. By uniqueness of the first eigenvalue for the above problem, it follows that

$$
B k_{\sqrt{B} L, a, b, c}(\lambda)=k_{L, a, \sqrt{B} b, B c}(\sqrt{B} \lambda)
$$

for all $L>0, B>0$ and $\lambda \in \mathbb{R}^{N}$. From the definition (9), one gets that, for any unit vector $e$ of $\mathbb{R}^{N}$,

$$
w_{L, a, \sqrt{B} b, B c}^{*}(e)=\sqrt{B} \times w_{\sqrt{B} L, a, b, c}^{*}(e)
$$

and Corollary 2.5 then follows from Theorem 2.2.

\section{References}

[1] D. G. Aronson and H. G. Weinberger. Multidimensional non-linear diffusion arising in population genetics. Adv. Math., 30(1):33-76, 1978.

[2] G. Barles and B. Perthame. Discontinuous solutions of deterministic optimal stopping time problems. RAIRO Mod. Math. Anal. Num., 21(4):557-579, 1987. 
[3] G. Barles and B. Perthame. Exit time problems in optimal control and vanishing viscosity method. SIAM J. Control Optim., 26(5):1133-1148, 1988.

[4] H. Berestycki and F. Hamel. Front propagation in periodic excitable media. Comm. Pure Appl. Math., 55(8):949-1032, 2002.

[5] H. Berestycki and F. Hamel. Generalized travelling waves for reaction-diffusion equations. In H. Berestycki, M. Bertsch, F. Browder, L. Nirenberg, L. Peletier, and L. Véron, editors, Perspectives in Nonlinear Partial Differential Equations: in Honor of Haïm Brezis, volume 446 of Contemporary Mathematics Series, pages 101-123, 2007.

[6] H. Berestycki, F. Hamel, and G. Nadin. Asymptotic spreading in heterogeneous diffusive excitable media. J. Funct. Anal., 255(9):2146-2189, 2008.

[7] H. Berestycki, F. Hamel, and N. Nadirashvili. The speed of propagation for KPP type problems. I - Periodic framework. J. Europ. Math. Soc., 7(2):173-213, 2005.

[8] H. Berestycki, F. Hamel, and N. Nadirashvili. The speed of propagation for KPP type problems. II - General domains. J. Amer. Math. Soc., 23:1-34, 2010.

[9] H. Berestycki, F. Hamel, and L. Roques. Analysis of the periodically fragmented environment model: II - Biological invasions and pulsating travelling fronts. J. Math. Pures Appl., 84(8):1101-1146, 2005.

[10] H. Berestycki and G. Nadin. Asymptotic spreading for general heterogeneous KPP equations. In preparation, 2010.

[11] L. A. Caffarelli, K.-A. Lee, and A. Mellet. Homogenization and flame propagation in periodic excitable media: the asymptotic speed of propagation. Comm. Pure Appl. Math., 59:501$525,2006$.

[12] M. El Smaily, F. Hamel, and L. Roques. Homogenization and influence of fragmentation in a biological invasion model. Disc. Cont. Dyn. Syst. A, 25:321-342, 2009.

[13] L. C. Evans. Periodic homogenization of certain nonlinear partial differential equations. Proc. Royal Soc. Edinburgh A, 120:245-265, 1992.

[14] L. C. Evans and P. E. Souganidis. A PDE approach to geometric optics for certain semilinear parabolic equations. Indiana Univ. Math. J., 38:141-172, 1989.

[15] R. A. Fisher. The wave of advance of advantageous genes. Ann. Eugenics, 7:335-369, 1937.

[16] M. Freidlin. On wave front propagation in periodic media. In M. Pinsky, editor, Stochastic analysis and applications, volume 7 of Advances in Probability and Related Topics, pages 147-166, 1984.

[17] M. Freidlin. Limit-theorems for large deviations and reaction-diffusion equations. Ann. Proba., 13(3):639-675, 1985.

[18] M. Freidlin and J. Gärtner. On the propagation of concentration waves in periodic and random media. Soviet Math. Doklady, 20:1282-1286, 1979. 
[19] J. Gärtner. Bistable reaction-diffusion equations and excitable media. Math. Nachr., 112:125-152, 1983.

[20] F. Hamel. Qualitative properties of monostable pulsating fronts: exponential decay and monotonicity. J. Math. Pures Appl., 89:355-399, 2008.

[21] F. Hamel, J. Fayard, and L. Roques. Spreading speeds in slowly oscillating environments. Bull. Math. Biology, 72(5):1166-1191, 2010.

[22] F. Hamel and L. Roques. Uniqueness and stability properties of monostable pulsating fronts. J. Europ. Math. Soc., to appear.

[23] S. Heinze. Homogenization of flame fronts. Preprint IWR, Heidelberg, 1993.

[24] S. Heinze, G. Papanicolaou, and A. Stevens. Variational principles for propagation speeds in inhomogeneous media. SIAM J. Appl. Math., 62:129-148, 2001.

[25] B Khouider, A Bourlioux, and A Majda. Parameterizing the burning speed enhancement by small-scale periodic flows: I. Unsteady shears, flame residence time and bending. Comb. Theory Modelling, 5:295-318, 2001.

[26] N. Kinezaki, K. Kawasaki, and N. Shigesada. Spatial dynamics of invasion in sinusoidally varying environments. Pop. Ecology, 48(4):263-270, 2006.

[27] N. Kinezaki, K. Kawasaki, F. Takasu, and N. Shigesada. Modeling biological invasions into periodically fragmented environments. Theo. Pop. Biology, 64(3):291-302, 2003.

[28] A. N. Kolmogorov, I. G. Petrovsky, and N. S. Piskunov. Étude de l'équation de la diffusion avec croissance de la quantité de matière et son application à un problème biologique. Bull. Univ. d'État Moscou, Sér. Internationale A, 1:1-26, 1937.

[29] P.-L. Lions and P. E. Souganidis. Homogenization of "viscous" Hamilton-Jacobi equations in stationary ergodic media. Comm. Part. Diff. Equations, 30:335-375, 2005.

[30] A. J. Majda and P. E. Souganidis. Large-scale front dynamics for turbulent reaction-diffusion equations with separated velocity scales. Nonlinearity, 7:1-30, 1994.

[31] G. Nadin. Travelling fronts in space-time periodic media. J. Math. Pures Appl., 92:232-262, 2009.

[32] G. Nadin. Some dependence results between the spreading speed and the coefficients of the space-time periodic Fisher-KPP equation. Preprint, 2010.

[33] G. Nadin. The effect of the Schwarz rearrangement on the periodic principal eigenvalue of a nonsymmetric operator. SIAM J. Math. Anal., 41(6):2388-2406, 2010.

[34] J. Nolen, M. Rudd, and J. Xin. Existence of KPP fronts in spatially-temporally periodic advection and variational principle for propagation speeds. Dynamics Part. Diff. Equations, $2: 1-24,2005$.

[35] J. Nolen and J. Xin. Reaction-diffusion front speeds in spatially-temporally periodic shear flows. SIAM J. Multiscale Model. Simul., 1:554-570, 2003. 
[36] J. Nolen and J. Xin. Asymptotic spreading of KPP reactive fronts in incompressible spacetime random flows. Ann. Inst. H. Poincaré, Analyse Non Linéaire, 26:815-839, 2009.

[37] J. Nolen and J. Xin. KPP fronts in 1D random drift. Disc. Cont. Dyn. Syst. B, 11:421-442, 2009.

[38] A. Okubo and S. A. Levin. Diffusion and Ecological Problems - Modern Perspectives. Second edition, New York: Springer-Verlag, 2002.

[39] R. Pinsky. Second order elliptic operator with periodic coefficients: criticality theory, perturbations, and positive harmonic functions. J. Funct. Anal., 129:80-107, 1995.

[40] N. Shigesada and K. Kawasaki. Biological Invasions: Theory and Practice. Oxford Series in Ecology and Evolution, Oxford: Oxford University Press, 1997.

[41] H. F. Weinberger. On spreading speeds and traveling waves for growth and migration in periodic habitat. J. Math. Biol., 45:511-548, 2002.

[42] F. Williams. Combustion Theory. Reading, MA, USA: Addison-Wesley, 1983.

[43] J. Xin. Front propagation in heterogeneous media. SIAM Review, 42:161-230, 2000. 